\title{
Estudio teórico sobre el efecto de la reología de las tintas en la resolución y problemática de impresión en los diseños cerámicos
}

\author{
G. PERIS-FAJARNÉS, P.B. SÁNCHEZ, P. LATORRE, M.J. PÉREZ, F. BRUSOLA \\ Grupo de Investigación en Tecnologías Gráficas. Universidad Politécnica de Valencia. Escuela Politécnica Superior de Gandia. \\ Ctra. Nazaret-Oliva, s/n. 46730 Grao de Gandia (Valencia) España.
}

\begin{abstract}
Los sistemas de impresión en la industria del pavimento y revestimiento cerámico permiten la realización de una adecuada transferencia del diseño a la pieza final. La evolución técnica de cada uno de estos procesos de impresión permite hacerlo casi con independencia del tipo de sistema que se utilice. Además, podemos decir que, en los últimos años, se ha llegado a un notable aumento de las posibilidades en cuanto a diseños en su forma y su calidad (entendida esta como aumento en la resolución). Las mejoras en cuanto a la precisión "teórica" de las técnicas suponen un aumento de problemas de impresión de importancia en el producto. Un aumento en la resolución de impresión supone la aparición de nuevos problemas en la estabilidad del tono producido por la mayor dificultad de control de punto de impresión. Este problema es especialmente grave en la cerámica donde se exige una homogeneidad tonal de todo el producto fabricado. En el presente artículo se describen las interacciones que existen entre las variables de diseño, los sistemas de impresión y las características físico-químicas de las tintas que afectan a la estabilidad de los diseños en pavimentos y revestimientos cerámicos. El objetivo es destacar la necesidad de controlar sistemáticamente los máximos factores posibles con el fin de reducir la variabilidad tonal productiva. El presente trabajo es parte de un proyecto de investigación centrado en la minimización de la variabilidad tonal en el sector cerámico financiado con fondos Feder-Cicyt.
\end{abstract}

Palabras clave: Cerámica, reología, diseño, resolución, ganancia de punto

Effect of the ink rheology in the resolution and printing impression problem in the ceramic designs. Theoretical study.

Printing systems at the flooring and covering tile industry allow the achievement of an adequate transference from design to the final piece. Technical evolution of each one of these systems permits to make it almost independently from the printing system used. Besides, it could be said that in the last few years it has arrived at an outstanding increase in the variety of designs in terms of definition and quality (being understood as an increase in the resolution). As far as the "theoretical" precision is concerned, they entail an increase in printing problems, being important in the product. A higher printing resolution implies the appearance of new problems in tone stability provoked by the higher difficulty in the control of the printing dot. This problem is specially grave in ceramics, where tone homogeneity of the product is required. In this paper interaction between variables related to design, as well as printing systems, and characteristics of physical and chemical properties of the dyes that affect stability of designs in ceramic flooring and covering tiles are described. The aim is to stand out the necessity to systematically control as many factors as possible in order to reduce production tone variability. This piece of work is part of a research project financed through FEDER-CICYT funds, focused on the minimisation of tone variability in the ceramic tile industry.

Key words: Ceramic, rheology, design, resolution, dot gain

\section{INTRODUCCIÓN}

En la Industria Cerámica el proceso de creación de un determinado modelo comienza en la fase de diseño, estableciendo las propiedades formales de los productos. Desde el punto de vista de un diseñador cerámico los aspectos más relevantes a tener en cuenta son: la innovación, la evolución tecnológica, el valor estético, la funcionalidad del producto y la adecuación a las características socioeconómicas y culturales de los usuarios. Una vez éste crea la imagen que se desea transmitir se descompone en colores. En este momento nos encontramos con la posibilidad de usar colores específicos para el diseño o bien utilizar una descomposición en cuatricromía, teniendo en cuenta que la cuatricromía hoy en día sólo se utiliza en sistemas de inyección de tinta y en determinadas flexográficas.
Desde la etapa de diseño a producción en planta, los técnicos han de tener en cuenta una multitud de parámetros y tomar decisiones al respecto. Las principales etapas a tener en cuenta son:

Modo de descomposición de colores, elección de esmaltes, engobes, pastas, etc.

Método de impresión de pruebas, modo de tramado (AM, FM), resolución y procesos intermedios (filmación, insolación, etc.).

Formulación y preparación de las tintas, engobes, esmaltes, etc.

- Proceso de transferencia del diseño a la línea (puesta a punto).

- Producción en planta. 
El modelo, una vez ha sido finalizado y aceptado en cuanto a su diseño, es decir, una vez que se decide fabricar, se lanza desde diseño a producción, debiendo pasar por una serie de fases (filmación, generación soportes intermedios, ajuste prensado, preparación tintas y esmaltes etc.). En cada una de dichas fases, se debe de disponer de toda la información necesaria para poder realizarse, es decir, desde diseño, el modelo debe de salir junto con toda la información necesaria para cada uno del resto de procesos implicados en su fabricación. El modelo, una vez se ha fabricado, exige un control al final de la línea basado habitualmente en una inspección visual pieza a pieza para la eliminación de piezas defectuosas o rotas y para la catalogación de cada modelo en submodelos que difieren en el tono de impresión o en su calibre.

En cuanto a defectos superficiales de las piezas, se puede asumir la posibilidad de que aparezcan defectos por roturas, marcas, roces y otras alteraciones imprevistas que podríamos clasificar como ocasionales, y en el caso en que se repitiesen de manera habitual, una inspección de la línea podría dar fácilmente con la causa pudiendo subsanarse. En cuanto a la variabilidad tonal, pese a ser un problema habitual en el sector, sigue siendo un elemento que exige de una clasificación, pues su origen no está, aparentemente localizado en un punto exacto o definido por un factor concreto. No obstante, dado que nos encontramos en un proceso industrial, aparentemente repetible, debería ser posible evitar dicha variación mediante el control de aquellos factores que intervienen directamente en aplicaciones relacionadas con el tono, es decir, en los factores que intervienen en la línea de esmaltado que pueden ser medidos. Es razonable pensar en que es posible controlar casi totalmente dichas fuentes de variación. De hecho, existen datos que apuntan al sistema de impresión como causa de variación del tono, en concreto, sólo teniendo en cuenta la serigrafía, ésta es la responsable de una variabilidad de aproximadamente un $50 \%$ en el tono (1-7) debido a los diferentes parámetros de impresión y preimpresión. Mediante la metodología planteada se reducirán los costes asociados a la clasificación por categorías, además de aumentar la calidad final del producto fabricado.

La implementación del conocimiento presentado está económicamente justificado, ya que los costes asociados a la no-calidad de las empresas azulejeras puede estimarse en un $22 \%$ de los costes totales (8), siendo los costes de prevención de tan sólo el $0.9 \%$. Teniendo en cuenta los datos anteriores y datos económicos del sector azulejero, donde puede indicarse que el potencial de producción de las empresas en el sector cerámico en España en 1999 era de 602 millones de metros cuadrados (9), produciéndose realmente en ese año dos billones de pesetas, ocupando detrás de Italia un papel de líder internacional en la producción mundial de azulejos (10). Siendo además, la zona de Castellón, la productora de $93.4 \%$ de la producción nacional, teniendo en su territorio al $80 \%$ de las empresas del sector. Hay que tener en cuenta que ha habido un crecimiento económico en los últimos años que ha estado asociado aun notable incremento de la calidad y los procesos de innovación tecnológica (11).

Por tanto, si se observan los datos anteriores, tanto económicos como de no-calidad, se puede admitir que la implementación de un tramado óptimo y una eficaz sistematización en el proceso de preimpresión está económicamente justificado debido a que cualquier tipo de mejora que se implante en el sector cerámico tendrá un impacto notable teniendo en cuenta el potencial de producción de las industrias.

El problema de la estabilidad del tono originado por la dificultad de control del depósito en la impresión cerámica, la discusión y análisis de las causas, el efecto del aumento de resolución de los sistemas de impresión cerámicos y el cómo poder abordar métodos de control sobre la variación del proceso es lo que se va a discutir y definir en el presente artículo.

\section{PROBLEMÁTICA DEL PROCESO DE IMPRESIÓN CERÁMICO. CONTROL DE LA GANANCIA DE PUNTO.}

Los sistemas mecánicos de impresión tan sólo pueden actuar mediante la opción de dejar o no dejar un depósito de tinta sobre el soporte cerámico, pudiendo, dependiendo del sistema que estemos trabajando, ajustar también la cantidad de tinta en un determinado depósito. Si el depósito es estable en cantidad y en forma para todos y cada uno de los puntos depositados en las diferentes aplicaciones supondrá una igualdad en las repeticiones de la impresión, y no supondrá un cambio de tono final, al menos producido por el sistema de impresión (debe recordarse que hay estudios ya citados que indican que más del $50 \%$ de la variabilidad tonal se debe directamente a la impresión en producciones realizadas por serigrafía plana). No obstante, hoy en día puede decirse que no es así. Una serie de factores que vamos a abordar a lo largo del artículo demuestran que la cantidad de depósito de los sistemas de impresión no es siempre igual, y además su variabilidad es mayor cuanto mayor es la resolución de la imagen que se desea aplicar o imprimir sobre las piezas. En Artes Gráficas ya se estableció el concepto extensible por completo al proceso de impresión cerámico definido como ganancia de punto, o diferencia entre el tamaño de punto diseñado y el finalmente obtenido en la impresión. En el caso de la industria cerámica, si bien la base del problema es la misma, y el concepto de ganancia de punto válido, existe un mayor número de interacciones y factores que pueden suponer una todavía mayor dispersión tonal debido, además de la propia variabilidad en el depósito, a las interacciones de éste con el resto de factores y variables del proceso de fabricación.

Si destacamos además la problemática de impresión derivada del soporte sobre el que se imprime, se puede comprender la mayor dificultad de control de la impresión en el sector cerámico. En el proceso cerámico se imprime sobre una superficie cruda (bizcocho), con temperaturas entre 40 y 80 grados centígrados, con piezas húmedas y con la necesidad de realizar impresiones superpuestas sin estar completamente secas las piezas y sin haber, de momento, posibilidad de utilizar sistemas de secado rápido entre las impresiones tal y como se hace en la industria gráfica mediante el uso de "Tintas UV" o tintas de secado por disparos de luz Ultra Violeta que precisamente se desarrolló para evitar dicho efecto. Estos factores uno a uno y combinados afectan, sin duda al proceso de impresión y al tono final de la pieza. Experiencias ya realizadas han demostrado un efecto claro de la temperatura sobre el tono final de la pieza impresa (45). Es necesario estudiar y medir las interacciones de todos y cada uno de las variables que intervienen en el proceso sobre los diferentes sistemas de impresión si se desea controlar y reducir totalmente la variabilidad tonal. De hecho existen numerosos trabajos ya realizados que relacionan las diferentes fases del proceso con el tono final de impresión. Una revisión y recopilación de dichos trabajos fue presentada recientemente en el Congreso Qualicer 2002 en forma de póster (57). 


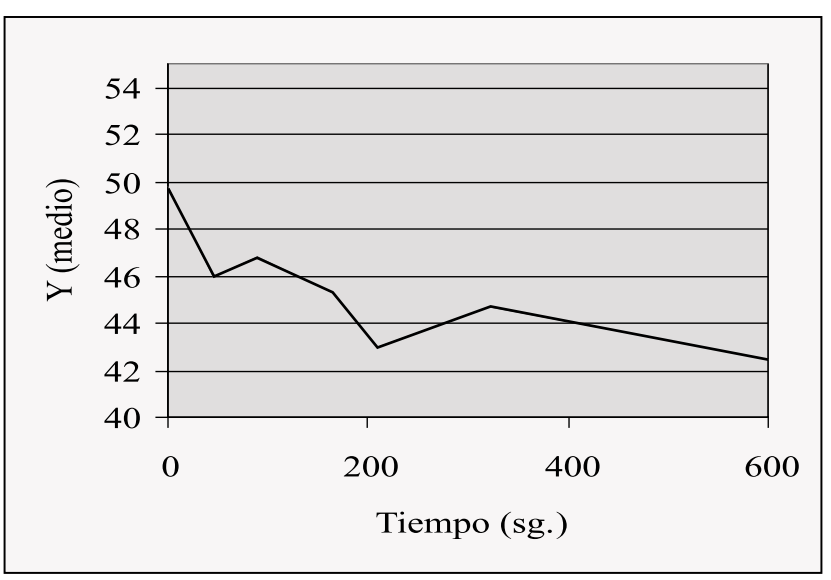

Gráfico 1. Efecto del tiempo transcurrido sobre el tono final de la pieza en un ensayo realizado en un proceso productivo por monococción y una impresión serigráfica con tinta negra sobre esmalte blanco. Gráfica que representa el valor de Y (CIE) sobre una serie de piezas impresas y cocidas. El tiempo se representa en segundos y el 0 corresponde con una entrada en el cabezal de impresión sin ninguna parada, y el 600 supone una parada de 10 minutos. La impresión sobre la que se midió se correspondía con una imagen homogénea de un nivel de gris tramado. Se muestra el efecto lineal que produce un retardo que pudiera producirse en una línea por una posible parada productiva. El retraso supone un enfriamiento de la pieza y un posible efecto secundario sobre la curvatura de esta (45).

\subsection{Ganancia de punto}

La ganancia de punto consiste en un aumento del tamaño del punto en el proceso de impresión como resultado del encharcamiento de la tinta debido a fenómenos físicos tales como la adherencia y fenómenos de capilaridad, y depende principalmente de la reología de la tinta, del soporte sobre el que se va a imprimir y del perímetro del punto de tramado (13). Un punto de menor dimensión puede aumentar mucho más su tamaño que uno de mayor dimensión, lo que indica que una forma de reducir la variabilidad tonal sería utilizar un punto suficientemente grande para que su ganancia sea pequeña, junto con un sistema de tramado que reduzca su efecto e incremente su gama de tonalidades.

Los diferentes fabricantes de maquinaria cerámicos al desarrollar los nuevos sistemas de impresión tratan de llegar a alcanzar la máxima definición en el diseño mediante un aumento de la resolución (mediante la impresión de un mayor número de puntos por centímetro lineal). Un aumento de la resolución conlleva un menor tamaño del punto de impresión generándose un problema de estabilidad tonal $(14,15)$. Existe, por tanto, una relación entre la definición de los sistemas de impresión y la problemática de estabilidad tonal. (16).

Dado que en la Industria Cerámica se tiende a una mayor definición, ello también va a conllevar, un mayor problema en el control del punto impreso, de la Ganancia de Punto. Para reducir o evitar este problema va a ser necesario un mucho mayor control de todos y cada uno de los factores de diseño e impresión que intervienen en el proceso, es necesario disponer del conocimiento y experiencia que relacione las variables y parámetros que se definen en diseño con los sistemas de impresión, sus limitaciones y particularidades.

\subsection{Tramado}

En el diseño de un determinado modelo, cuando se decide aplicar sobre la pieza cerámica, debe realizarse el proceso de transformación de la imagen digital a una imagen binaria (opaca-translúcida) en la que las zonas opacas corresponden a los lugares en los que el pigmento, del color determinado, va a quedar depositado en la impresión. El proceso intermedio que genera dicha imagen es el tramado. Precisamente el tramado puede permitirnos definir en parte la geometría, tamaño y otros factores del fututo depósito, y es por ello uno de los pasos que pueden permitir controlar adecuadamente el depósito de tinta que se aplica a la pieza. Como ya se ha comentado, los sistemas de impresión tan sólo pueden tomar la decisión de dejar o no un depósito de tinta en un determinado punto, el agrupamiento de un mayor o menor número o la modificación del tamaño de estos puntos es lo que permite variar las tonalidades impresas que se observan. Es por tanto necesario conocer bien el sistema de tramado y escogerlo correctamente en función del sistema de impresión e incluso del diseño que se desee aplicar.

Los sistemas de tramado más extendidos en la Industria Gráfica en general son la trama convencional (AM, en el que la cantidad de punto a lo largo de la trama es constante y la simulación de tono continuo se consigue mediante la variación del tamaño de punto), la trama estocástica (FM, en el que el tamaño del punto es constante y la simulación del tono continuo se consigue con la variación de la concentración del punto, es decir, con la variación de la cantidad de punto) y la trama híbrida (que es una combinación de las dos anteriores, siendo recomendable para los trabajos de alta calidad donde es necesario conservar el detalle en las altas luces, mantener la riqueza de los tonos medios, y perfecta definición en las sombras) (17).

Mediante el tramado AM (tramado tradicional), los puntos no son todos iguales y, por ejemplo, en serigrafía conforme aumenta el tamaño de los puntos no lo hace el tejido y se pierde en escala de grises intermedia y en resolución (18). Esto no ocurre con el tramado FM, donde si su usa de manera correcta, no solo garantiza que todos los puntos aparezcan, sino que además, reduce considerablemente otros problemas como el moaré.

Uno de las formas o tipos más conocidos de tramado FM es el generado mediante la técnica de "Error Diffusion". Este sistema o método tuvo y sigue teniendo una importante particularidad, y es precisamente que trata de corregir las diferencias entre lo que se debe imprimir y lo que realmente se puede. Su funcionamiento básico puede explicarse con un breve ejemplo: cuando una impresora en blanco y negro necesita en un punto imprimir un nivel de gris, la decisión a tomar en cada uno de los puntos de impresión es sólo si deja o no una gota impresa, es decir, la impresora sólo dejará una gota de tinta si dicho gris esta mas cerca del negro que del blanco, es decir con un $51 \%$ de negro. Si aplicamos el método de error diffusion (19), el siguiente punto que la impresora tenga que imprimir se decidirá utilizando el error de puntos adyacentes, es decir, un punto con un $51 \%$ de negro solo puede imprimirse con un $100 \%$, y su error por exceso se repartiría proporcionalmente entre todos los puntos que le rodean, $y$, de esta forma, se corrigen las diferencias entre lo que se debe imprimir y se puede imprimir. Este sistema modificado es el que se usa en casi todas las impresoras de inyección de tinta y ha supuesto una notable mejora en la calidad que se obtiene de dichos dispositivos. Curiosamente, y pese a disponerse ya de 
experiencias muy satisfactorias en otros campos de las Artes Gráficas, en la Industria cerámica, no se utiliza de manera habitual. El uso de dicho procedimiento en la industria cerámica causaría una reducción de pérdidas de puntos de impresión, un control de ganancia de punto y una casi total desaparición de riesgo de moaré, indirectamente ello provocaría, a su vez, una reducción de problemas de impresión, de variaciones de tonalidad y del tiempo necesario entre la creación del modelo y su preparación para su producción. De todos modos, la aplicación de estos tipos de tramado para los diferentes sistemas de impresión, pese a las aparentes ventajas que teóricamente aporta, no es tan evidente, ya que en la práctica un cálculo erróneo de una resolución de trama aleatoria (FM) puede perjudicar mucho la definición de la imagen que fuera elaborada mediante una trama tradicional (AM).

El hecho de la no-utilización del tramado FM en el sector cerámico es debido principalmente a que los sistemas de tramado comerciales no permiten el control del tamaño de punto, además, no ha estado, desde un principio, insertado dentro del lenguaje Postcrip y por ello no es una opción que pueda fácilmente configurarse para un RIP estándar; no es una opción automatizada dentro de las opciones de tramado de imagen existentes dentro de los programas comerciales, y por último, exige de una mayor laboriosidad en la preparación de los fotolitos. No obstante, se dispone de metodologías $\mathrm{y}$ experiencias que garantizan y facilitan su uso de manera precisa y correcta (P9600915).

\subsection{Sistemas de impresión: resolución de tramado y mínimo punto de tamaño teórico}

Una vez definido el sistema de tramado, se hace necesario preparar el sistema de impresión (que debe haberse definido con anterioridad a la elección del tipo de tramado). Actualmente, los diferentes sistemas de impresión cerámicos comerciales podemos agruparlos básicamente en estas cuatro categorías:

Serigrafía plana y rotativa, cuyo método de impresión consiste en hacer pasar tinta a través de un tejido con ayuda de una espátula.

Huecograbado, basado en la utilización de un rodillo de silicona en el que se han realizado cavidades que definen le diseño que se desea transferir.

Flexografía, técnica basada en la transferencia de tinta por contacto mediante el relieve de un rodillo impresor.

Impresión digital, que consiste en hacer pasar la tinta a través de una boquilla que la pulveriza, se desvían las gotas de modo que impacten en la pieza en diferentes puntos para crear el diseño.

La elección del sistema de impresión se determinará en función del modelo, numero de tintas, resolución del diseño, relieves, etc. Desde el punto de vista de capacidad (resolución) de cada uno de los dispositivos de impresión, nos encontramos con datos que exigen una breve discusión para poder ser comparados, y que se indican en la tabla (Tabla 1). Los datos que se indican sobre la resolución de impresión, obtenidas de las diferentes casas comerciales en el caso de Huecograbado, Flexografía e Impresión digital, responden a una resolución límite binaria, en cambio, la resolución, en el caso de la Serigrafía plana, se refieren a resolución, una vez tramada la imagen, dado que la resolución binaria, en este caso depende del fotolito intermedio. Por ello, para poder realizar una comparación entre los sistemas, deberíamos referir- nos al límite del tamaño de punto mínimo teórico, y en este sentido, también es necesario aclarar las diferencias entre la Inyección de Tinta y el resto de sistemas. En cuanto al tamaño mínimo de punto, se refiere al límite teórico, siempre que la tinta se comporta perfectamente y su reología se encuentre perfectamente definida, tratándose por tanto de condiciones ideales. En cuanto al número de colores del sistema de inyección, el sistema trabaja siempre a una resolución máxima lineal en sentido horizontal, siendo la resolución vertical dependiente de la velocidad de impresión, el sistema, además por basarse en una cuatricromía, dispone, por punto de impresión un mayor número de colores (que el resto de sistemas también pueden obtener mediante impresiones consecutivas).

En cuanto a la obtención de la resolución real, podemos afirmar que las tintas en serigrafía están mucho más estudiadas, más adaptadas a la técnica y su comportamiento, y por tanto los valores teóricos máximos están más contrastados y probados que en el resto de los sistemas de impresión. En huecograbado y flexografía, actualmente, se producen con mayor frecuencia fenómenos de encharcamiento, aumentando el punto de impresión y, por tanto la ganancia de punto. Este fenómeno se acentúa para el caso de huecograbado, ya que utilizan tintas con bajo límite de fluencia y baja viscosidad, lo que produce que la gota transferida del rodillo de silicona a la baldosa se extienda con facilidad sobre la pieza.

TABLA I.- COMPARATIVA DE LA RESOLUCIÓN DE TRAMADO Y DEL MÍNIMO TAMAÑO DE PUNTO TEÓRICO PARA SERIGRAFÍA, HUECOGRABADO, FLEXOGRAFÍA E INYECCIÓN DE TINTA. Para serigrafía se ha tomado como referencia una pantalla de 250 hilos / cm y una resolución de 40 Líneas $/ \mathrm{cm}$. Para el sistema de inyección de tinta, pese a no ser necesario el tramado de la imagen, se ha establecido una resolución de tramado similar con el fin de poder tener una referencia para la comparación. Con estos valores se podría obtener hasta 36 niveles de gris y un tamaño de punto teórico de $40 \mu \mathrm{m}$ (condiciones para una pantalla con una excelente calidad). En huecograbado y flexografía, en condiciones similares a la serigrafía podríamos llegar a obtener 225 niveles de gris y un tamaño de punto teórico de $17 \mu \mathrm{m}$. Para inyección de tinta, el diámetro de la boquilla determina el tamaño de punto mínimo teórico que es de $100 \mu \mathrm{m}$. En inyección de tinta, se considera una resolución horizontal y vertical iguales, habiéndose obtenido el número de colores considerando que el sistema funciona siempre con cuatricromía.

\begin{tabular}{|c|c|c|c|}
\hline \multicolumn{4}{|c|}{ RESOLUCIÓN Y TAMAÑO DE PUNTO MÍNIMO $(\mu \mathrm{m})$} \\
\hline SERIGRAFÍA & HUECOGRABADO & FLEXOGRAFÍA & INYECCIÓN DE TINTA \\
\hline $40 \mathrm{~L} / \mathrm{cm}$ & $600 \mathrm{ppcm}$ & $600 \mathrm{ppcm}$ & $180 \mathrm{ppcm}$ \\
\hline 250 hilos $/ \mathrm{cm}$ & $40 \mathrm{~L} / \mathrm{cm}$ & $40 \mathrm{~L} / \mathrm{cm}$ & (Si se considerase $40 \mathrm{~L} / \mathrm{cm}$, \\
\hline 36 niveles de gris & 225 niveles de gris & 225 niveles de gris & $\begin{array}{c}\text { obtendriamos } 4096 \text { colores } \\
\text { posibles por punto) }\end{array}$ \\
\hline $40 \mu \mathrm{m}$ & $17 \mu \mathrm{m}$ & $17 \mu \mathrm{m}$ & $55 \mu$ \\
\hline
\end{tabular}

Existen diferentes estudios cuyo principal objetivo es la estandarización de un método que permita asegurar una sistematización en las condiciones de trabajo y de todos los parámetros indicados en la elaboración de la pantalla, independientemente de que se realice en la propia empresa o esta sea subcontratada, entre los que cabe destacar el mostrado por Alicer (20) y el realizado en la Universidad Politécnica de Valencia. En la Universidad Politécnica de Valencia se ha desarrollado una patente (P9600915) con título: "Procedimiento para el cálculo de tramado tipo Diffusion o Dithering para la elaboración de pantallas de serigrafía", en el 
cual se describe el método para el cálculo de la resolución de tramado destinado a la insolación de pantallas de serigrafía para maximizar la eficacia de la insolación, evitar la aparición de moaré, reducir el porcentaje de pérdida de punto, controlar al mínimo el tamaño de punto impreso y maximizar el número de niveles de gris imprimibles. Además, debido a que la frecuencia óptima de tramado se obtiene mediante un método visual de análisis de imagen, el cálculo es independiente del equipo de impresión y, en principio, los resultados obtenidos podrían ser aplicables al resto de sistemas de impresión (flexografía, huecograbado, etc.). Mediante esta tecnología las empresas pueden disponer de un proceso digital estable, ya que se pueden eliminar con ciertas garantías la fuente de variación debida a la digitalización de la imagen y al proceso de transformación en una imagen binaria en la elaboración del clisé.

\section{INTERACCIONES Y FACTORES A ANALIZAR EN EL PROCESO DEL DISEÑO A LA IMPRESIÓN.}

El problema de estabilidad tonal proviene principalmente del tamaño del punto de impresión y por tanto de la resolución. Para los diferentes sistemas de impresión en la Industria Cerámica la problemática es diferente, ya que ni la maquinaria ni las tintas se comportan del mismo modo.

En la producción de pavimentos y revestimientos cerámicos existen diferentes alternativas comerciales para realizar la transferencia del diseño a las piezas. La serigrafía es hasta hoy, con mucha probabilidad, la más usada en sus dos variantes, plana y rotativa, pero además, y desde hace relativamente poco tiempo, el uso de la flexografía, el huecograbado y el actual sistema en desarrollo de inyección de tinta, son elementos que están siendo utilizados con gran eficacia.

La mayoría de las técnicas de impresión de la Industria Cerámica provienen de la adaptación de sistemas de impresión en Artes Gráficas, donde han tenido un largo tiempo de desarrollo. Estos sistemas, se han traído a la industria cerámica con el claro objetivo de mejorar el rendimiento productivo y aumentar en la diversidad de diseños en la producción. Además, mediante los nuevos sistemas de impresión (flexografía, huecograbado e inyección de tinta entre otros), se pretende evitar el límite físico de resolución que tiene la serigrafía, debido praecisamente a la hilatura del tejido. Hoy, podemos afirmar que el aumento en la definición, queda limitado por las características de las tintas en cerámicas, de compleja adaptación por su naturaleza y composición química. Además, debe apuntarse la dificultad añadida derivada de la necesidad de garantizar una total igualdad tonal en toda la producción, condición que no se exige en igual medida en la industria gráfica.

Precisamente, el conocimiento de las características de las tintas y de los parámetros que deben de ser controlados en los sistemas de impresión, es el único medio para conseguir esa importante garantía de repetibilidad productiva necesaria en la fabricación del pavimento y revestimiento cerámico. Las características, desde lo general a lo particular se definen a continuación.

\subsection{Tintas cerámicas}

Las tintas cerámicas que se utilizan en la actualidad en cerámica son suspensiones de sólidos concentradas de com- posición variable en un líquido de naturaleza orgánica (generalmente naftalenos y parafinas) o hidrosoluble (lo más comunes hechos a base de óxidos de etileno y derivados) denominado vehículo (21). Los sólidos son básicamente fritas y pigmentos. Las fritas y los pigmentos que se usan se encuentran previamente molturados para que el tamaño de partícula de los sólidos sea el adecuado para cada uno de las técnicas de impresión. Los vehículos son en su mayoría polietilenglicoles de bajo peso molecular y los ligantes que se utilizan cuando se seca la tinta son los denominados fijadores (disoluciones concentradas de alcohol de polivinilo y/o acetato de polivinilo en agua). Actualmente se incorporan aditivos mezclados con los vehículos para funciones específicas.

\subsection{Serigrafía}

En Serigrafía, debido a la cantidad de tintas que existen en el mercado, así como a la variación de tonalidad que se produce en la cocción, es de suma importancia el conocimiento químico (22) y reológico de los pigmentos y pastas que se utilizan (23). El comportamiento de las tintas durante su aplicación en la fase de impresión está regulado por las características reológicas del producto: viscosidad, gradiente de velocidad, diámetro de partícula, etc. $(24,25)$. La viscosidad debe ser lo suficientemente baja cuando atraviesa la pantalla y lo suficientemente alta en reposo para permitir una elevada definición en los puntos impresos (26). Estas características son típicas de fluidos pseudoplásticos con elevado límite de fluencia (27). Para ajustar la viscosidad de la tinta, hace falta llegar a un compromiso para evitar que la tinta se disperse en el fondo y permitir que se impriman los puntos más pequeños en las partes claras de la imagen. La viscosidad cambia a lo largo del tiempo de impresión, es decir, cambia su valor al ser extendida mediante la rasqueta, al pasar por los orificios de la pantalla, etc. (28), por tanto será de suma importancia mantenerla constante antes de la impresión y durante ésta.

Las fritas y los pigmentos, componentes de las tintas cerámicas, se encuentran previamente molturados para que el tamaño de la partícula sea el adecuado para la luz de malla del tejido de la pantalla y, por tanto, el tamaño del punto que se desea reproducir. Según estudios realizados (29), los pigmentos deben ser como máximo un tercio del tamaño de la abertura de la malla, sin embargo, cada vez más se tiende a obtener materiales de tamaño de grano más fino, de forma controlada y con una adecuada reología para obtener una mayor definición del punto y, debido a que las pantallas cada vez más los orificios son de menor tamaño. El tamaño de las partículas debe ser el adecuado para que atraviese la pantalla y no la obture, por tanto, se debe reducir el tamaño de los componentes de las tintas por debajo de 40 $\mu \mathrm{m}$ aproximadamente, evitando además los aglomerados que aumentan el tamaño de partícula. Cabe tener en consideración dos aspectos asociados al tamaño de partícula, por un lado el brillo de la decoración final que está relacionado con el tamaño de las agrupaciones (a mayor agregación menor brillo, según estudios realizados (30)), y por otro, el estado de dispersión de las partículas que influye sobre el comportamiento de la tinta en la aplicación serigráfica (influye sobre la viscosidad, ya que es muy sensible tanto al tamaño de partícula como a su dispersión) y sobre las características finales del recubrimiento (intensidad del color obtenido) (31). 
TABLA II.- VALORES CARACTERÍSTICOS DE TINTAS SERIGRÁFICAS.

\begin{tabular}{|c|c|c|c|}
\hline Luz de malla $\left(\mathbf{n}^{\mathbf{0}}\right.$ hilos & 36 & 68 & 77 \\
\hline Densidad $\left(\mathbf{g} / \mathbf{c m}^{\mathbf{3}}\right)$ & $1.7-1.9$ & $1.6-1.7$ & $1.6-1.7$ \\
\hline$\eta_{\gamma=\mathbf{0 . 1 s}}{ }^{-1} \mathbf{( c P )}$ & $\approx 10^{5}$ & $10^{4}-10^{5}$ & $10^{4}-10^{5}$ \\
\hline$\eta_{\gamma=1000 \mathbf{s}^{-1}} \mathbf{( c P )}$ & $\approx 10^{3}$ & $\approx 10^{3}$ & $\approx 10^{3}$ \\
\hline $\mathbf{D}_{\mathbf{5 0}}(\mu \mathbf{m})$ & $4.5-5.5$ & $4.5-5.5$ & $4.5-5.5$ \\
\hline
\end{tabular}

D: diámetro de partícula

$\eta$ : viscosidad

\begin{tabular}{|c|c|c|c|c|}
\hline Repeticiones & $\begin{array}{l}\text { una vez } \\
\text { por diseño }\end{array}$ & $\begin{array}{l}\text { una vez } \\
\text { por fotolito } \\
\text { (tantos fotolitos } \\
\text { como tintas) } \\
\end{array}$ & $\begin{array}{c}\text { una vez por } \\
\text { pantalla (tantas } \\
\text { pantallas como } \\
\text { tintas) } \\
\end{array}$ & $\begin{array}{|lr|}\text { una vez por pieza (cada pieza } \\
\text { es impresa por tantas } \\
\text { pantallas como tintas tenga el } \\
\text { diseño) }\end{array}$ \\
\hline $\begin{array}{l}\text { PREPARACIÓN } \\
\text { (pantalla y cabezal) }\end{array}$ & $\begin{array}{l}\text { creación } \\
\text { del diseño } \\
\text { descomposición } \\
\text { decolores } \\
\text { tramado }\end{array}$ & $\begin{array}{l}\text { generación } \\
\text { del fotolito }\end{array}$ & \begin{tabular}{|l|}
$\begin{array}{l}\text { elaboración } \\
\text { de pantalla } \\
\text { emulsionado } \\
\text { insolación }\end{array}$ \\
$\longrightarrow$ \\
revelado \\
secado
\end{tabular} & \begin{tabular}{|l||} 
colocación de la pantalla \\
en el cabezal de impresión
\end{tabular} \\
\hline IMPRESIÓN & & & & $\begin{array}{c}\text { centrado y ajuste } \\
\text { impresión: fase de carga } \\
\quad \text { aase de impresión }\end{array}$ \\
\hline
\end{tabular}

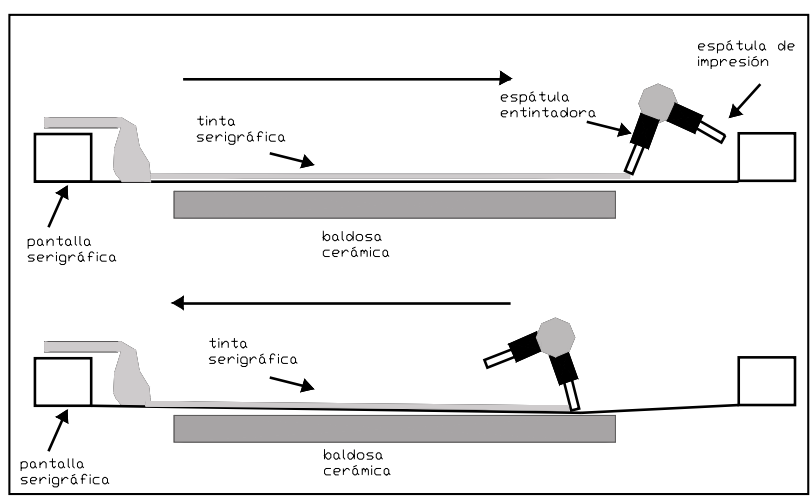

Figura 1.- Proceso de Impresión Serigráfica. El proceso de impresión puede estructurarse en dos fases, una fase de carga, donde una de las espátulas reparte la tinta por la pantalla, y una fase de impresión donde una espátula presiona el tejido con la tinta y obliga a esta a pasar a través del tejido. La presión de la espátula en la fase de impresión debe ser lo suficientemente alta como para que la pantalla contacte con la superficie a imprimir. Además, cuando la imagen que se desea obtener está formada por varios colores o tintas, el proceso anterior se realiza una vez por cada color, y usando una pantalla diferente para cada uno y será imprescindible realizar un centrado entre las impresiones para obtener una imagen nítida y un registro óptimo.

Las propiedades de las tintas deberán ser estables durante las condiciones de aplicación para garantizar la repetitibilidad de las piezas, y a lo largo de la producción. Habrá que tener en cuenta la variación con la temperatura, ya que un aumento de la temperatura reduce la viscosidad de los vehículos (32)). La perfección de una óptima suspensión serigráfica requiere de un equilibrio tanto de las características reológicas como del resto de propiedades que inciden en la calidad final del producto (tiempo de secado de la tinta, permeabilidad a los gases, etc.).

Una vez la tinta ha pasado los controles de laboratorio y se encuentra perfectamente definida y estudiada para un determinado modelo, ha de tenerse en cuenta una serie de paráme- tros a la hora de controlar el proceso y de reducir la variabilidad tonal (33): las variables de las pantallas de serigrafía (proceso de preparación-proceso de preimpresión, características de la malla y el marco, etc.) (34-36) y las variables del propio proceso de impresión en la línea de esmaltado (posición de la pantalla y la espátula, variables mecánicas, etc.) (37).

El proceso de preimpresión determina en gran medida la calidad del diseño final de la pieza (38), ya que es la pantalla la portadora de la imagen a transferir. El proceso de preimpresión consiste principalmente en la elaboración de la pantalla de impresión mediante una serie de procedimientos definidos pero con una escasa automatización y sistematización. El proceso de elaboración de las pantallas exige un control total de todas las variables físicas (espesor, luz de paso, tensión, etc.) (39) ya que cada uno de las etapas en el proceso puede afectar a la calidad final de las piezas $(40,41)$.

En cuanto al proceso de impresión en planta, destacar que es necesario realizar un pormenorizado ajuste de todos los parámetros del cabezal serigráfico (42) en la línea de esmaltado y controles rutinarios durante la producción que garanticen que las propiedades de las tintas permanecen constantes, mediante la medida de densidad, viscosidad y gramaje depositado en la pieza (43). La colocación de la pantalla en el cabezal es un proceso rutinario, pero será necesario controlar todas las variables del cabezal (posicionamiento, sujeción, centrado de la pantalla, fuera de contacto, ángulo y afilado de espátulas y presión sobre la pantalla, y para el caso de rotativa ángulo de torsión de la pantalla, entre otros) (44-46), además de regular la velocidad de impresión, caudal de serigrafía, etc., es decir, las variables mecánicas e hidrodinámicas, a lo largo de la producción. Una vez controlados todos los parámetros tendremos la garantía de que podemos poner en marcha un modelo garantizado la repetitibilidad del diseño $y$, por tanto, optimizando la variabilidad tonal.

El proceso de serigrafía está controlado hoy en día, en cuanto al conocimiento de las limitaciones y posibilidades, y los equipos de diseñadores de las empresas solventan con su experiencia las limitaciones de este campo. En el departamento de diseño se enmascaran los defectos y en el de químicos se desarrollan tintas estables y de comportamiento conocido suavizando los problemas asociados a la variabilidad del cabezal de serigrafía, debido a esto la serigrafía sigue funcionando bien, pero todavía queda mucho por investigar.

\subsection{Huecograbado}

En el proceso de impresión por huecograbado cabe analizar las principales variables que afectan al diseño final de las piezas: los parámetros del proceso de fabricación del aplicador (preimpresión), las propiedades y comportamiento de las tintas (47) y los parámetros de la propia impresión en planta (48).

La transferencia del diseño al rodillo impresor se lleva a cabo a partir de un soporte magnético que envía la información al láser que incide sobre el rodillo de silicona realizando las cavidades que conforman el diseño. Dependiendo de la resolución con la que se haya definido la transferencia del diseño al rodillo, el láser efectúa los disparos más o menos intensos y los alveolos (cavidades) que ser producirán en el rodillo serán diferentes. Dependerá de las características de los alveolos y de la resolución seleccionada se obtendrán distintas descargas de tinta sobre las baldosas, definiéndose el diseño de la pieza. El potencial existente de este sistema de 
impresión no está hoy en día explotado completamente debido a que la preparación del rodillo supone hoy en día un alto coste para las empresas de fabricación de maquinaria y un alto precio de compra para los productores cerámicos (49).

Las tintas que se utilizan en este sistema de impresión deben poseer un bajo límite de fluidez para que puedan con facilidad abandonar las cavidades del rodillo, y una baja viscosidad en el intervalo de gradientes de velocidad de trabajo. Este comportamiento es típico newtoniano (los fluidos newtonianos cumplen la ley de Newton y su viscosidad no debe variar con el tiempo de cizalla, suelen ser fluidos con estructura molecular sencilla) y con escasa tixotropía (la viscosidad aparente disminuye con el tiempo de aplicación, al finalizar el cizallamiento sólo se recupera la viscosidad inicial después de un tiempo más o menos largo), además de esfuerzos mínimos de fluencia (yield point) (50). El porcentaje de color con respecto a la serigrafía incrementa, ya que para cilindros con diseños a baja resolución $(0.4 / 45)$ se trabaja alrededor de $50-60 \%$ y para alta definición (100 puntos/ $\mathrm{cm}$ ) alrededor de un $80-90 \%$ de color (51), datos obtenidos de producciones concretas.

TABLA III.- VALORES CARACTERÍSTICOS DE TINTAS PARA HUECOGRABADO.

\begin{tabular}{|c|c|}
\hline Densidad $\left(\mathbf{g} / \mathbf{c m}^{\mathbf{3}}\right)$ & $1.4-1.6$ \\
\hline$\eta_{\gamma=\mathbf{0 . 1}}{ }^{-\mathbf{1}}(\mathbf{c P})$ & $1000-5000$ \\
\hline$\eta_{\gamma=\mathbf{1 0 0 0 s}}{ }^{-1}(\mathbf{c P})$ & $\approx 100$ \\
\hline $\mathbf{D}_{\mathbf{5 0}}(\mu \mathbf{m})$ & $5-6$ \\
\hline
\end{tabular}

D: diámetro de partícula

$\eta$ : viscosidad

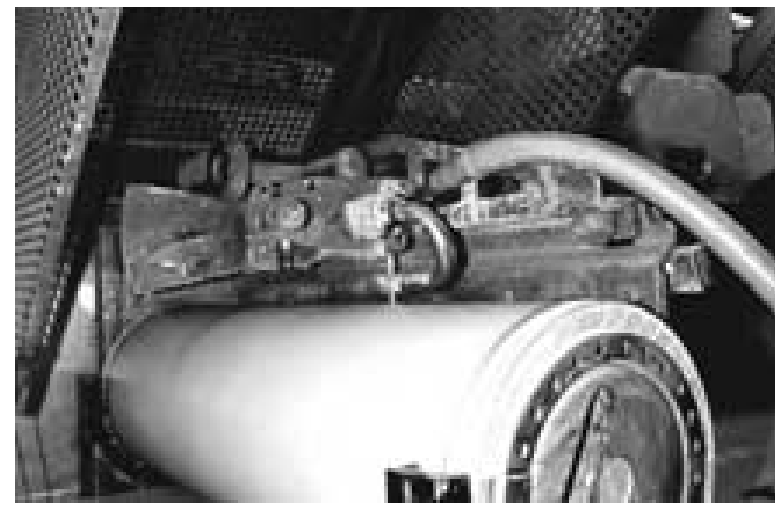

Figura 2.- Proceso de impresión por huecograbado.

En el proceso de impresión en planta es necesario ajustar y controlar las variables del equipo de impresión, tales como el ángulo y la presión de la espátula entre otras, que influyen en la cantidad de tinta que llena las cavidades del rodillo impresor y por tanto en el tamaño final del punto de impresión.

Será necesario, para garantizar una óptima impresión, un comportamiento reológico óptimo y estable a lo largo del tiempo y del proceso productivo cerámico.

\subsection{Flexografía}

En la fabricación de pavimentos y revestimientos cerámicos mediante flexografía existen diferencias significativas en las propiedades de las tintas (composición química y compor- tamiento reológico) con respecto a la serigrafía y al huecograbado. La transferencia de la tinta del aplicador a la baldosa se realiza por contacto del relieve del rodillo impresor mediante la dosificación de la tinta de modo constante y homogéneo mediante un rodillo dosificador tramado que actúa como tintero (52). El comportamiento reológico de la tinta viene determinado por el porcentaje de color, que puede llegar a ser de un $80 \%$ para obtener tonalidades intensas. El comportamiento de las suspensiones en flexografía es ligeramente pseudoplástico, con valores de viscosidad a diferentes gradientes de velocidad prácticamente constantes, con bajo límite de fluencia y apenas tixotropía. La viscosidad ha de ser relativamente alta a bajos gradientes de velocidad para que ni se desprenda del relieve del polímero antes de que entre en contacto con la baldosa ni se deslice por el rodillo dosificador. La viscosidad de las tintas para este sistema tiene un rango amplio de trabajo sin que se produzcan problemas de variabilidad tonal, principalmente a bajas temperaturas de trabajo, de 5 a $20^{\circ} \mathrm{C}$. La intensidad de la tinta y por tanto de los puntos impresos depende principalmente de la densidad de la tinta, mientras que la definición depende de la base de la suspensión. Otra de las características que interesa conocer y controlar en la tinta es la tensión superficial, ya que cuando sea baja provocará que la tinta moje más el esmalte y al separarse el rodillo de la baldosa se produzca una mejor transmisión de la tinta y se consiga una mayor intensidad en cada punto impreso.

TABLA IV.- VALORES CARACTERÍSTICOS DE TINTAS PARA APLICAR POR FLEXOGRAFÍA.

\begin{tabular}{|c|c|}
\hline Densidad $\left(\mathrm{g} / \mathrm{cm}^{3}\right)$ & 1.5 \\
\hline$\eta_{\gamma=0.1 \mathrm{~s}-1}(\mathrm{cP})$ & $\approx 5000$ \\
\hline$\eta_{\gamma=100 \mathrm{~s}-1}(\mathrm{cP})$ & $\approx 1000$ \\
\hline $\mathrm{D}_{50}(\mu \mathrm{m})$ & $3.5-4.5$ \\
\hline
\end{tabular}

D: diámetro de partícula

$\eta$ : viscosidad

\begin{tabular}{|c|c|c|c|}
\hline$\overbrace{\text { Secuencia }}^{\text {Repeticiones }}$ & $\begin{array}{l}\text { una vez } \\
\text { por diseño }\end{array}$ & $\begin{array}{c}\text { una vez por rodillo } \\
\text { (tantos rodillos } \\
\text { como tintas) }\end{array}$ & $\begin{array}{l}\text { una vez por pieza } \\
\text { (cada pieza es impresa por tantos } \\
\text { rodillos como tintas tenga el diseño) }\end{array}$ \\
\hline $\begin{array}{l}\text { PREPARACIÓN } \\
\text { (rodillo impresor) }\end{array}$ & \begin{tabular}{|l|} 
creación \\
del diseño \\
descomposición \\
de colores \\
tramado
\end{tabular} & \begin{tabular}{|c|}
$\begin{array}{c}\text { generación } \\
\text { del rodillo } \\
\text { impresor }\end{array}$ \\
$\begin{array}{l}\text { (mediante láser se } \\
\text { realizan las cavidades) }\end{array}$ \\
\end{tabular} & $\begin{array}{l}\text { colocación del rodillo en } \\
\text { la estación de huecograbado }\end{array}$ \\
\hline IMPRESIÓN & & & $\begin{array}{l}\text { trado y ajuste } \\
\text { ón: la tijtas se deposita en la parte superior del rodillo } \\
\text { la rasqueta fuerza el llenado de las cavidades } \\
\text { mediante contacto se transfiere la tinta a la baldosa }\end{array}$ \\
\hline
\end{tabular}

Figura 3.- Proceso de impresión mediante flexografía.

El equipo consta de un rodillo liso, que ha sido construido alrededor de un núcleo metálico recubierto de una envolvente flexible que facilita la absorción de irregularidades de las piezas cerámicas evitando roturas por el exceso de presión. Sobre este rodillo se fija una lámina de polímero que presenta una zona en relieve (realizada mediante grabación láser digital), correspondiente al dibujo que se desea reproducir. Una vez la lámina se encuentra dispuesta sobre el rodillo se pone en contacto con otro rodillo dosificador tramado que actúa como tintero y dosifica de modo constante y homogéneo la cantidad de tinta que es transferida al fotopolímero, de modo que la parte en relieve de su superficie se impregna en la misma. Posteriormente, cuando el rodillo, debido a su giro, entra en contacto con la baldosa, transfiere a esta la tinta de que es portador, produciéndose la estampación. 
Los equipos basados en esta técnica se encuentran en proceso de investigación, desarrollo e implantación industrial, siempre teniendo como referencia la maquinaria ya implantada en otros sectores como es la Industria Gráfica (53).

\subsection{Impresión Digital}

La impresión digital por chorro de tinta continuo consiste en hacer pasar la tinta cerámica a través de una boquilla pulverizándola, formándose un chorro de pequeñas gotas que se desvían, conformando el diseño de la pieza a decorar. La principal ventaja que presenta este sistema es la no-utilización de ningún soporte intermedio donde se encuentre el diseño, favoreciendo la agilidad del proceso y teóricamente aumentando la calidad final debido a la no-transmisión de errores producidos en la transferencia fotolito-aplicador y aplicadorpieza (54).

La calidad de impresión depende en gran medida de las propiedades de la tinta, tales como viscosidad, conductividad, tensión superficial, ph y la estabilidad del flujo de tinta (55), características generales de este sistema en los sectores en los que se presenta. Más concretamente, en la Industria Cerámica, se debe tener muy en cuenta el tamaño de partícula, ya que los componentes son cristales inorgánicos, y por tanto, el tamaño de la boquilla debe ser mayor que en el resto de las aplicaciones de inyección de tinta utilizadas en otros sectores, siendo por tanto de hasta $100 \mu \mathrm{m}$, generando un mayor tamaño de punto con respecto a los otros sistemas de impresión. Debido a la erosión que producen los cristales cerámicos, la boquilla deberá poseer una mayor resistencia al desgaste y a la corrosión. A diferencia del resto de sistemas, la tinta deberá poseer susceptibilidad eléctrica, ya que para generar el diseño sobre la pieza, las gotas se desvían mediante un sistema eléctrico, y para ello es necesario añadir aditivos con esta capacidad a la base de la suspensión. Teniendo en cuenta todos los factores citados, será necesario llegar a un compromiso entre el tamaño de los cristales, la dispersión de la tinta y la viscosidad para obtener un registro óptimo mediante este sistema (56).

\section{CONCLUSIONES.}

En el proceso de diseño, el aumento de resolución de tramado de un determinado modelo, supondrá siempre un mayor número de "tonos" de dicho modelo frente al caso en que dicho modelo se realizase con una resolución inferior.

Para la preparación del aplicador, es necesario utilizar un procedimiento sistemático y en el que las relaciones entre el aplicador (sea cual fuere el sistema de impresión), la forma y resolución de tramado sean óptimas, constantes y repetibles. Para ello tanto las variables ambientales como aquellas que se definen desde diseño deben ser siempre las mismas. La preparación del soporte intermedio (rodillo de flexografía, huecograbado o pantalla serigráfica), debe ser realizada en laboratorios de ambiente controlado y con maquinaria calibrada; así como el almacenamiento de reactivos y materiales que intervienen en el proceso deben también estar controlado. Es necesario realizar una medición de tiempos de proceso, de parada y de reposo de cada uno de los pasos que se realiza sobre el aplicador, debiéndose además documentar dichos datos.

El proceso de impresión, debe realizarse manteniendo las variables constantes a lo largo de toda la producción. Tal y como se ha comentado, existen experiencias realizadas que demuestran cómo, pese a mantener constantes las variables de impresión dependientes del cabezal, alteraciones de, por ejemplo temperaturas debidas a paradas de 30 segundos, producen un cambio de tono detectable según los criterios habituales de clasificación. El control tanto de variables del cabezal como del entorno y piezas que reciben la impresión (temperatura, humedad, características del esmalte, etc.) son, evidentemente, factores a mantener constantes a lo largo de la impresión. Del mismo modo que habrá que tener en cuenta el proceso de impresión superpuesta o también denominado decoración sobre decoración.

De los diferentes sistemas de impresión, el huecograbado frente a la serigrafía plana o a la flexografía, es el que puede, teóricamente, obtener una mayor resolución de impresión con una relativa mayor estabilidad tonal. No se disponen de suficientes datos para poder comparar dicho proceso con la Impresión directa por Inyección de Tinta.
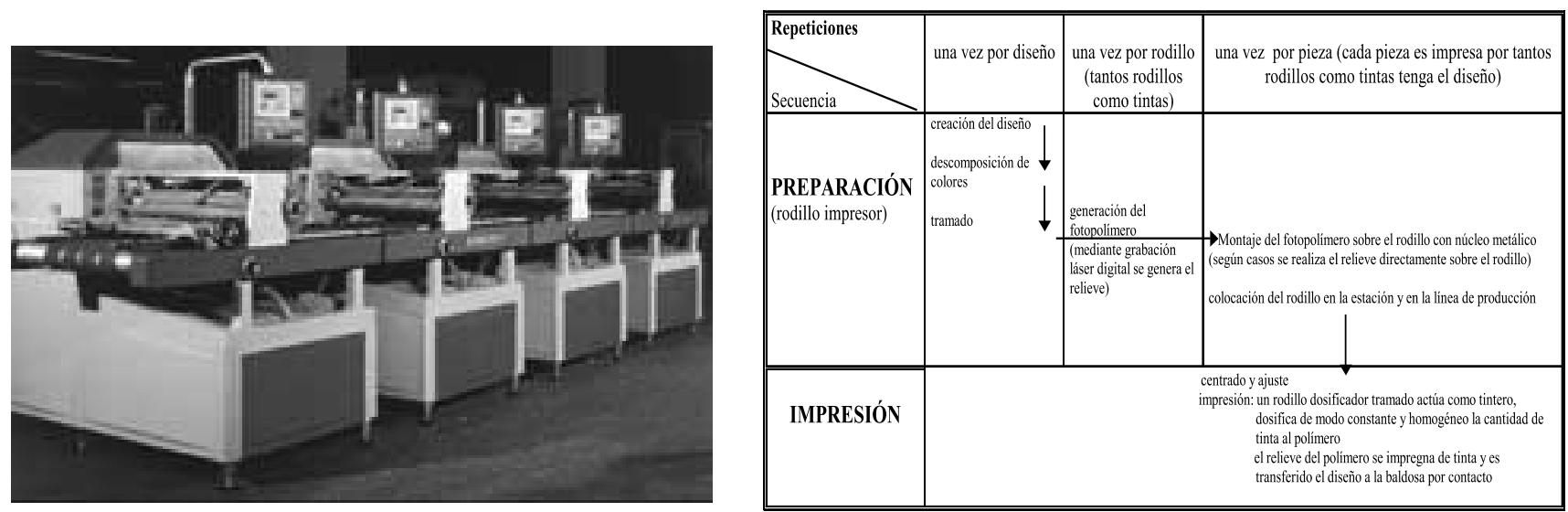

Figura 4.- Impresión digital. La impresión por chorro de tinta consiste en hacer pasar la tinta a través de una boquilla que la pulveriza formando un chorro de pequeñas gotas que deben alcanzar la pieza a decorar. Se trata de desviar las gotas de modo que impacten en la pieza en diferentes puntos para crear el diseño deseado. Esto puede combinarse con el movimiento de la pieza pudiéndose obtener decoraciones más complejas. Las gotas se desvían mediante un sistema eléctrico y si lo que se desea es que no se depositen se dejan sin carga y se recogen mediante un sistema de recuperación, actuando sobre el sistema carga - no carga se obtiene el dibujo definitivo. 


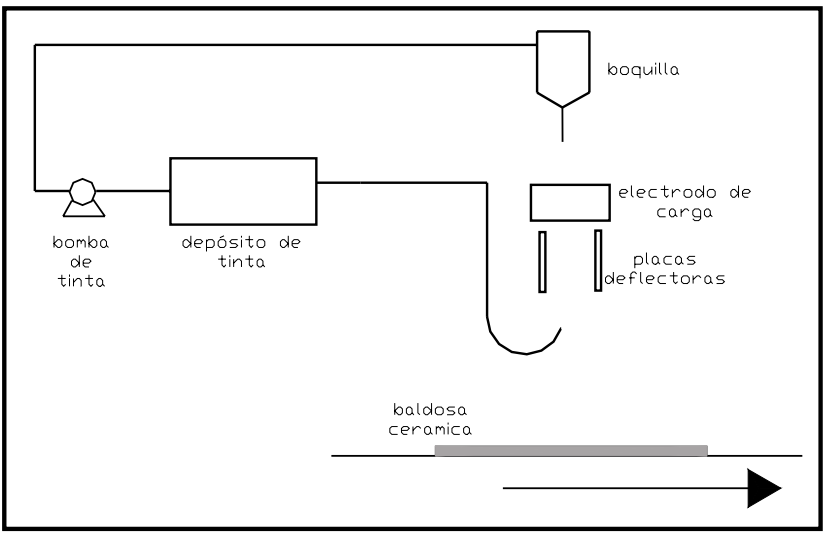

Se han estudiado los más utilizados sistemas de impresión cerámicos analizando la influencia de los diferentes parámetros reológicos en el tamaño del punto de impresión y por tanto en la resolución. Destacaremos las principales cuestiones a tener en cuenta los técnicos y los fabricantes del sector cerámico.

\section{Serigrafía:}

- Es importante controlar la viscosidad, densidad y gramaje depositado de la tinta a lo largo de la producción como indicativo de una correcta impresión de las piezas como consecuencia de una estabilidad reológica, y dado que las variaciones de temperatura afectan notablemente a la viscosidad, es fundamental mantener la temperatura de las tintas constante. Así mismo, se debe mantener una constante agitación de las tintas para evitar agregados que producen una disminución del brillo de la pieza, además la intensidad del color final está relacionada con la dispersión de partículas.

- El tamaño de fritas y pigmentos debe ser el adecuado según la luz de malla del tejido ya que determina el tamaño del punto de impresión. Se recomienda que el $95 \%$ de las partículas tengan un tamaño inferior a un tercio de la luz de malla.

- Dado que es el sistema más antiguo, su implementación y relación entre resolución de tejido y de diseño permiten obtener calidades excelentes. Existe una limitación debida al número de hilos, no siendo habitual superarse en la industria gráfica y cerámica los 200 hilos por centímetro. Resoluciones de malla superiores exigen hilos mas finos (lo que hace más débil al tejido) y produce mayores problemas de obturación de las mallas.

Huecograbado:

- Las tintas en huecograbado deben poseer un bajo límite de fluidez para que pueda abandonar con facilidad las cavidades del rodillo y una baja viscosidad en el intervalo de gradientes de velocidad de trabajo. Comportamiento típico newtoniano y con escasa tixotropía. Debido al bajo límite de fluidez que poseen las tintas y a la baja viscosidad, con mayor probabilidad se producen fenómenos de encharcamiento y aumento del punto de impresión.

- Se acentúa el problema de la sedimentación de las tintas debido principalmente al bajo límite de fluidez de éstas y a la baja viscosidad de trabajo, por lo que es prácticamente imprescindible el uso de agitación constante e uniforme para garantizar condiciones de operación estacionarias.

- Dado que el depósito que se produce en la impresión es menor que en la impresión serigráfica, se debe de subir considerablemente el porcentaje de color para obtener tonalidades equivalentes. Algunas experiencias apuntan a un incremento muy notable en el porcentaje de color respecto a la serigrafía cifrándolos entre un 50 y un $90 \%$. (51)

- La resolución del huecograbado viene dado por los disparos que efectúa el láser definiéndose las cavidades. Dependiendo de las características de los alveolos y de la resolución seleccionada que se obtengan las diferentes descargas sobre las baldosas. El tamaño final del punto de impresión viene además definido por el ángulo y la presión de la espátula, que influye en la cantidad de tinta que llena las cavidades del rodillo impresor.

- Del mismo modo que en flexografía, el contacto pieza rodillo puede suponer que una pequeña parte del esmalte quede retenido en el rodillo y pase al depósito de tinta. Dado que el proceso se repite a lo largo de toda la producción, el control del efecto que dicho esmalte pueda tener sobre la tinta es importante.

Flexografía:

- Los valores de la viscosidad son prácticamente constantes, aunque es necesario trabajar con valores de viscosidad altos para que la tinta no se desprenda del relieve del polímero. La densidad de la tinta es la que define la intensidad de la tinta y de los puntos impresos y la definición depende de la base de la suspensión.

- La tensión superficial habrá que controlarla ya que determina la intensidad del punto impreso debido a que produce que la tinta moje más o menos el esmalte y se realice una mejor transmisión de la tinta a la pieza.

- Para este sistema de impresión, se pueden utilizar diámetros de partícula bajos de hasta $3.5 \mu \mathrm{m}$. Ello permite imprimir teóricamente tamaños de punto menores, siendo para ello necesario un riguroso control de la estabilidad de la tinta.

- Al igual que para huecograbado, generalmente se necesita una mayor intensidad en los colores para obtener resultados similares a los obtenidos por impresión serigráfica. Un de las empresas fabricantes de este tipo de sistemas de impresión (CREAPRINT) ha apuntado como dato la necesidad de aumentar el porcentaje de color en un $80 \%$ frente a uan impresión serigráfica.

Inyección de tinta:

- Los principales parámetros que hay que controlar y que determinan la calidad final son: viscosidad, conductividad, tensión superficial, ph y estabilidad del flujo de la tinta.

- Mediante la adición y control de aditivos se genera susceptibilidad eléctrica a las tintas necesaria para una correcta transmisión del diseño.

- El tamaño de partícula debe ajustarse al de la boquilla, que se están empezando a fabricar de hasta $100 \mu \mathrm{m}$ (27), determinando un mayor tamaño de punto impreso.

- Será necesario para un registro óptimo regular: el tamaño de los cristales, la dispersión de la tinta y la viscosidad. La resolución de impresión es constante horizontalmente y verticalmente variable dependiendo de la velocidad de impresión (siendo, habitualmente esta resolu- 
ción vertical igual o menor que la horizontal). Existen dispositivos que permiten superar los 600 puntos por centímetro de resolución horizontal. La calidad y definición impresa, dependerán mucho de los algoritmos de tramado de la imagen que utilice el sistema.

\section{BLIBLIOGRAFÍA.}

1. F. Negre, A. Moreno, E. Sánchez, et al., "Factores que influyen sobre la variabilidad de la tonalidad de baldosas cerámicas", Ponencia presentada al XXXIV Congreso Anual de la Sociedad Española de Cerámica y Vidrio, L’Alcora (Castellón), 14-17 de septiembre, 1994 (No publicada).

2. D. Scher, "Process color, cd printing", Screen Printing, may, $46-54$ (1996).

3. Instituto de Tecnología Cerámica (Castellón), Keraben S.A., Informe $n^{\circ}$ C930504: "Observación y análisis de varios clisés", junio 1993.

4. Instituto de Tecnología Cerámica (Castellón), Keraben S.A., Informe $n^{\circ}$ C930311: "Plan de actuaciones para la disminución de la dispersión de tonalidad durante la producción de azulejos por monococción", mayo 1993.

5. J.L. Amorós, L. Díaz, S. Giménez, V. Sanz, “Comportamiento reológico de las suspensiones de esmalte. Influencia de las características de la suspensión", Técnica Cerámica nº214 384-398 1993.

6. F. Negre, V. Sanz, S. Giménez, J.V. Agramunt, J.E. Martí, “Estado actual de la técnica de decoración de baldosas cerámicas". Ponencia presentada al XXXIV Congreso Anual de la Sociedad Española de Cerámica y Vidrio. L’Alcora (Castellón), 14-17 de septiembre de 1994 (No publicada).

7. Instituto de Tecnología Cerámica (Castellón), Keraben S.A., Informe n ${ }^{\circ}$ C930504: "Observación y análisis de varios clisés", junio 1993.

8. G. Peris Fajarnés, "Análisis cuantitativo de variables de impresión serigráfica que afectan a cambios de tono en piezas serigrafiadas", EN: V Congreso Mundial de la Calidad del Azulejo y del Pavimento Cerámico (QUALICER). Castellón: Cámara Oficial de Industria Comercio y Navegación, 265-281 1998.

9. P. Corma, J. F. Dávila, QPT-Consulting, "Evaluación de los costes de no-calidad del sector español de pavimentos y revestimientos cerámicos", EN: IV Congreso Mundial de la Calidad del Azulejo y del Pavimento Cerámico (QUALICER). Castellón: Cámara Oficial de Industria Comercio y Navegación, 381-398 1996.

10. I. Gil, J.J. Guarch y C. Andrés, "La industria cerámica de la Comunidad Valenciana en el ámbito nacional y europeo", Bol. Soc. Esp. Cerám. Vidrio, 38 [2] 133-141 (1999)

11. M. Regueiro, E. Sánchez, V. Sanz y E. Criado, "Cerámica Industrial en España", Bol. Soc. Esp. Cerám. Vidrio, 39 [1] 5-30 2000.

12. F. Ferrando, J.C. Orta, A. Moreno, V. Sanz, M.J. Orts, R. De Lemus, "Desarrollo de color y aparición de tonalidades en piezas de pavimento extruido esmaltado, tipo rústico", Bol. Soc. Esp. Cerám. Vidrio, 38 [6] 471-476 (1998).

13. "Euroescale. Un estándar a seguir", CMYK (Publicación técnica de AIDO para el sector de las Artes Gráficas), febrero de 2001.

14. Mark A. Coudray, "Causes and corrections of dot gain press", Screen Printing, agosto 18-26 1996

15. Claypole, T.C. Gethin, D.T. Danias, “ Dot gain ink transfer behaviour in the screen printing process", Infosurface coatings international, may 1997.

16. M. G. Langdon, "Pigment physics and their effect on printing inks", Jocca-surface coatings internacional, (10), pag. 431, 1993

17. Mark A. Coudray, “Interpreting imaged moire: funding the source before you go to press", Screen Printing, april, pag. 44-48, 1996.

18. "Tramas", CMYK (Publicación técnica de AIDO para el sector de las Artes Gráficas), febrero de 2001

19. R. Floyd, L. Steinberg, "An adaptative algorithm for spatial gray scale", In Society for Information Display 1975, Symposim Digest of Technical Papers, pg. 36, 1975.

20. AICE. Dispositivo para el control de pantallas serigráficas. Patente Española P9400337.

21. L. Francisco Pitta, "Veículos Serigráficos e Produtos Químicos Auxiliares", Cerâmica Industrial, 4(1-6) Janeiro/Dezembro, 1999

22. O. Ruiz, G. Monrós, F. Sanmiguel, "Relación entre las características magnéticas y la calidad de los pigmentos cerámicos", XL Congreso de la Sociedad Española de Cerámica y Vidrio, noviembre de 2000

23. C. Palmonari, A. Tenaglia, E. Rastelli, A. Albertazzi, R. Fornaciari, "Rheological study to test a new formulation of silk screen paint", Bol. Soc. Esp. Cerám. Vidrio, 39 [5] 627-630 (2000)

24. "Tecnologías de impresión: flexografía", Alabrent n 146, abril 1997.

25. L. F. Miller, "Screenability and Rheology", Solid State Technology 54-60 octubre 1974.

26. J.L. Amorós, L. Díaz, S. Giménez, V. Sanz, “Comportamiento reológico de las suspensiones de esmalte. Influencia de las características de la suspensión", Técnica Cerámica n²14, 384-398 1993

27. A. Moreno, "Adecuación de las propiedades de tintas y esmaltes a los sistemas de aplicación y técnicas decorativas", EN: VI Congreso Mundial de la Calidad del Azulejo y del Pavimento Cerámica (QUALICER). Castellón: Cámara Oficial de Industria Comercio y Navegación, 2000

28. T. X. Liang, W. Z. Sun, M. B. Tian, Y. H. Wang and H. D. Li, "The influence of the Characteristies of the Metal Particle in a Thick-Film Ink on its Screen Printing Aptitude", Surface Coatings International (5) 224-227 1996.
29. Sefar Inc. Switzerland, " ¡El procedimiento de impresión universal!

30. P. Negre, E. Sánchez V. Sanz A Gozalbo D. Alegre, A Carceller "Estimación del grado de dispersión de las tintas serigráficas". EN: IV Congreso Mundial de la Calidad del Azulejo y del Pavimento Cerámico (QUALICER). Castellón: Cámara Oficial de Industria Comercio y Navegación, 287-305 1996.

31. Albert Kosloff M.A., "Screen Printing Techniques", ST Publications inc., ISBN 0911380-52-3, 1994

32. R. Marín, A. Carratala, J. Miravet, S. Casares, M.J. Alfonso, P. Negre, et al, "Influencia del vehículo en el comportamiento reológico de las tintas serigráficas", EN: IV Congreso Mundial de la Calidad del Azulejo y del Pavimento Cerámico (QUALICER). Castellón: Cámara Oficial de Industria Comercio y Navegación, 755-758 1996.

33. J. Peñalver, V. Martí, J. Portolés, et al., "Estudio de las variables de control de aplicación serigráfica y su influencia sobre la dispersión de tonalidades en baldosas. EN: IV Congreso Mundial de la Calidad del Azulejo y del Pavimento Cerámico (QUALICER). Castellón: Cámara Oficial de Industria Comercio y Navegación, 323-335 1996.

34. M. Monzó, E. Bou, E. Sánchez, L. Gargallo, C. Arrébola, "Ensayos para la caracterización de pantallas serigráficas", Bol. Soc. Esp. Cerám. Vidrio, 40 [1] 17-24 (2001).

35. F. Negre, A. Moreno, S. Giménez, et al., "Caracterización de pantallas serigráficas utilizadas en la decoración de baldosas cerámicas". EN: XXXVI Congreso Nacional de Cerámica y Vidrio: libro de resúmenes. Arganda del Rey, Madrid: Sociedad Española de Cerámica y Vidrio, 1321996.

36. ZBF Equipo técnico, "El buen uso de los tejidos", En Serigrafía julio/agosto 25-29 1992.

37. S. Bermúdez, P. Corma, M. Gómez, R. Vaquero et al, “Influencia de las variables de serigrafiado sobre la presencia de tonalidades en baldosas cerámicas". EN: V Congreso Mundial de la Calidad del Azulejo y del Pavimento Cerámico (QUALICER). Castellón: Cámara Oficial de Industria Comercio y Navegación, Poster 97991998.

38. A. Peyskens, "Fundamentos técnicos de la realización de pantallas para serigrafía", Appiano Gentile: Saati, 1991.

39. G. Peris Fajarnés, Mariano Alcañiz Raya, "Introducción a la Serigrafía", En Serigrafía mayo - junio 1997.

40. Instituto de Tecnología Cerámica (Castellón), Keraben S.A., Informe n ${ }^{\circ}$ C 930121 "Procedimiento de trabajo para determinar la robustez de un modelo frente a la dispersión de tonalidades", abril 1993.

41. F. Martinez, "Variaçao de tonalidade: Design e Fotolitos", Cerâmica Industrial, 4(1-6) Janeiro/Dezembro, 1999.

42. S. Jones, "Fabricación de pantallas para un registro óptimo", En Serigrafía, noviembre/diciembre 14-17 1994

43. P. Corma, G. Bonet, A. Longedo, J. E. Martí, Y. Monsonís, “Estudio de la variabilidad en el grado de precisión de las máquinas de serigrafiado", EN: V Congreso Mundial de la Calidad del Azulejo y del Pavimento Cerámico (QUALICER). Castellón: Cámara Oficial de Industria Comercio y Navegación, Poster pag. 71998

44. G. Peris Fajarnés, "La serigrafía en el mundo de la producción cerámica", En Serigrafía, enero/febrero ${ }^{\circ} 541997$.

45. G. Peris Fajarnés, "Análisis de los parámetros de impresión serigráfica que afectan a la variación de tono obtenido en azulejos producidos por monococción", Tesis Doctoral, Universidad Politécnica de Valencia, 1997.

46. G. Peris Fajarnés, Pedro Latorre Carmona, "Serigrafía Cerámica. Manual de procedimiento orientado a la reducción de tonalidades", Grupo de Investigación en Tecnologías Gráficas. Universidad Politécnica de Valencia, Editorial Librería Politécnica 2001.

47. A. Peyskens, "Parámetros referentes a la fabricación serigráfica que afectan a la calidad de impresión", En Serigrafía, marzo/abril 1992.

48. J.A. Martínez, J. Cabedo, S. Jiménez, R. Flors, "Diseño de tintas optimizadas para decoración mediante la técnica de huecograbado". EN: XL Congreso Anual de la Sociedad Española de Cerámica y Vidrio. Onda (Castellón), libro de resúmenes pag. 79, 8-11 de noviembre de 2000.

49. Franco Stefani, "El futuro del proceso de esmaltado", System s.p.a. Italia, EN: V Congreso Mundial de la Calidad del Azulejo y del Pavimento Cerámico (QUALICER). Castellón: Cámara Oficial de Industria Comercio y Navegación, 57-65 1998.

50. "Tecnologías de impresión: Huecograbado", Alabrent (Revista Informativa del papel y las Artes Gráficas) n 147 mayo 1997.

51. Katia Canicossa, Dino T. Parisi e Fábio Ferraço, "Tecnologia Avançada para a Decoraçao de Revestimientos Cerâmicos", Cerâmica Industrial, 4(1-6) Janeiro/Dezembro, 1999.

52. Decoroil Productos Químicos, “Decoroil: vehículos para decoración mediante rodillos huecograbados", Cerámica Información n ${ }^{\circ} 269$ 77-79 noviembre de 2000.

53. Cretaprint, S.L., "Rotativa flexográfica: el futuro ya está presente", Técnica Cerámica n ${ }^{\circ} 280138-1402000$.

54. "Tecnologías de impresión: flexografía", Alabrent (Revista Informativa del papel y las Artes Gráficas) n ${ }^{\circ} 146$ abril 1997.

55. Jordi Magre, Antonio López, “La impresión digital: los sistemas, los soportes y los consumibles ideales", III Congreso Nacional de Serigrafía, Tampografía e Impresión Digital, Sevilla 2000.

56. M. G. Langdon, “ None impact printing”, Jocca-surface coatings internacional (10) pag. 4311993.

57. G. Peris-Fajarnés, E. Escuder, P.B. Sánchez, M.J. Pérez, P. Latorre, “ Revisión de estudios y referencias sobre los parámetros que pueden producir variabilidad tonal y defectos superficiales en la producción de azulejos y pavimentos cerámicos",VII Congreso Mundial de la Calidad del Azulejo y del Pavimento Cerámico, Qualicer 2002, Castellón 2002.

Recibido: 03.08.01

Aceptado: 13.05 .02 